\title{
First Results from the Parkes Multibeam High-velocity Cloud Survey
}

\author{
M. E. Putman ${ }^{1}$ and B. K. Gibson ${ }^{2}$ \\ ${ }^{1}$ Research School of Astronomy of Astrophysics, Australian National University, \\ Weston Creek PO, Weston, ACT 2611, Australia \\ putman@mso.anu.edu.au \\ 2 Center for Astrophysics and Space Astronomy, University of Colorado, \\ Campus Box 389, Boulder, CO 80309, USA \\ bgibson@casa.colorado.edu \\ Received 1998 November 9, accepted 1999 February 4
}

\begin{abstract}
With the onset of the Hi Parkes All-Sky Survey (HIPASS), a new view of the high-velocity cloud (HVC) distribution in the southern sky is being revealed. The dense spatial sampling and unbiased coverage of HIPASS gives it multiple advantages over previous surveys of the southern sky. Detailed views of the clouds' structure and large mosaics of the Hi sky allow us to link individual concentrations to larger structures (such as the Magellanic Clouds and the Galaxy), providing pictorial clues as to the origins of HVCs. It is clear that HVCs cannot be uniformly assigned a single origin scenario, and must be categorised appropriately.
\end{abstract}

Keywords: Galaxy: halo — Magellanic Clouds — ISM: structure

\section{Introduction}

High-velocity clouds (HVCs) are concentrations of neutral hydrogen which have velocities forbidden by simple models of Galactic rotation. Their velocities, with respect to the local standard of rest (lsr), typically fall in the range of $\left|v_{\text {lsr }}\right| \approx$ $80-400 \mathrm{~km} \mathrm{~s}^{-1}$, depending on the direction of observation.* Despite covering $\sim 37 \%$ of the sky (Murphy, Lockman \& Savage 1995), a consensus has yet to be reached on their origin. Several models have been proposed, both Galactic (e.g. supernova shells, warped structure in the disk, Galactic fountain infall/outflow) and extragalactic (e.g. remnants of the formation of the Local Group, tidally-disrupted material from the Magellanic Clouds or other dwarf galaxy companions), but theory alone has not been able to discriminate between these scenarios (and as we discuss here, ascribing the entire HVC population to one in particular would most likely be a mistake). Wakker \& van Woerden (1997) provided a timely review of the subject, both from an observational and theoretical point of view. In principle, observational tests can be devised which can discriminate between Galactic and extragalactic models, but to date, the implementation of such tests (e.g. direct distance determinations, metallicities, or emission measure properties) has proven difficult. Each of these observational tests depends on an understanding of the spatial and kinematical structure of HVCs, and while such high-quality $\mathrm{H}_{\mathrm{I}}$ data do exist in the north (e.g. Hulsbosch \& Wakker 1988; Hartmann \& Burton 1997), the southern sky has not enjoyed the same status.

To date the most complete investigation in the south is due to Bajaja et al. (1985, hereafter B85), who sampled the sky on a $2^{\circ} \times 2^{\circ}$ grid with the IAR $30 \mathrm{~m}$ at Villa Elisa. Next year should see the release of the updated Villa Elisa Southern Sky Survey (VESSS, Morras et al. 1999), the southern analog to the Leiden-Dwingeloo Survey (LDS, Hartmann \& Burton 1997), but for now one is restricted to the B85 survey for southern HVCs (and indeed, this was the data source for the southern half of the canonical catalogue of HVCs by Wakker \& van Woerden 1991). The B85 spatial sampling and beam size is wholly unsatisfactory for unveiling the presence of fine-scale structure and any large population of Compact HVCs (henceforth, CHVCs). In the subsequent sections, we present results from the first complete, fully sampled, southern sky survey for HVCs. Our initial results reveal intricate detail in the spatial structure of southern HVCs, a large population of undiscovered CHVCs, and filamentary connections between previously assumed discrete clumps.

\section{Observations and Data Reduction}

The Hi Parkes All-Sky Survey (HIPASS) is a blind Hi survey of the southern sky $\left(\delta \leq 0^{\circ}\right)$, over the velocity range -1200 to $12,700 \mathrm{~km} \mathrm{~s}^{-1}$ (StaveleySmith 1997). It therefore encompasses the Milky

\footnotetext{
* There is a well-known north-south hemisphere asymmetry in the HVC velocity distribution, such that the maximum $\left|v_{\text {lsr }}\right|$ seen in the north is $\sim 450 \mathrm{~km} \mathrm{~s}^{-1}, \sim 100 \mathrm{~km} \mathrm{~s}^{-1}$ greater than the maximum seen in the south.
} 
Table 1. HIPASS survey parameters versus those of B85 and VESSS

\begin{tabular}{cccc}
\hline Parameter & HIPASS & B85 & VESSS \\
\hline Telescope & Parkes $64 \mathrm{~m}$ & IAR $30 \mathrm{~m}$ & IAR $30 \mathrm{~m}$ \\
Sky & $\delta<20^{\circ}$ & $\delta<-15^{\circ}$ & $\delta<-25^{\circ}$ \\
HPBW & $15^{\prime} \cdot 5$ & $34^{\prime}$ & $34^{\prime}$ \\
Grid & $\sim 6^{\prime}$ & $120^{\prime}$ & $30^{\prime}$ \\
$\Delta v\left(\mathrm{~km} \mathrm{~s}^{-1}\right)$ & $26 \cdot 4$ & 16 & 8 \\
$T_{B}(\mathrm{~K})(5 \sigma)$ & $0 \cdot 045(5$ scans $)$ & $0 \cdot 125$ & \\
& $0 \cdot 1(1$ scan $)$ & & \\
$N_{\mathrm{HI}}\left(\mathrm{cm}^{-2}\right)(5 \sigma)$ & $1 \cdot 6 \times 10^{18}(5 \mathrm{scans})$ & $4 \cdot 6 \times 10^{18}$ & \\
$\left(\Delta v=20 \mathrm{~km} \mathrm{~s}^{-1}\right)$ & $3 \cdot 6 \times 10^{18}(1 \mathrm{scan})$ & & \\
\hline
\end{tabular}

Way, the Magellanic Clouds, and the more distant Universe. The survey is conducted with the Parkes $64 \mathrm{~m}$ radio telescope equipped with a multibeam receiver, a focal-plane array of 13 feeds set in a hexagonal grid. The spectrometer has 1024 channels for each polarisation and beam, with a channel spacing of $13 \cdot 2 \mathrm{~km} \mathrm{~s}^{-1}$. The survey is conducted by actively scanning the telescope in $8^{\circ}$ strips of declination, and displacing adjacent scans in RA such that sub-Nyquist sampling is achieved. The complete survey will scan the sky five times, with an effective integration time of $\sim 5$ minutes per sky point, corresponding to an rms brightness temperature sensitivity of $\sim 9 \mathrm{mK}$. The data presented in this paper only incorporate the first set of scans (which still Nyquist sample the sky), corresponding to an rms sensitivity of $\sim 20$ $\mathrm{mK}$.

The data were reduced using a modified version of the standard aips ++ based HIPASS reduction software (which was designed for imaging discrete Hi sources) (Barnes et al. 1998). To recover the extended structure of HVCs, we used the entire $8^{\circ}$ scan to calculate the bandpass correction for a particular channel and polarisation. Several statistical methods were tested, with the chosen method a compromise between recovering larger structures and the increasing variation in the correction for adjacent scans. The method chosen breaks each $8^{\circ}$ scan into five sections, finds the median flux in each section and uses the minimum of the five medians for the bandpass correction. This greatly increases our ability to recover large scale structure, except in areas where the emission fills more than $90 \%$ of a given scan (e.g. near the Galactic Plane). To minimise the effect of the ripple from Galactic $(\mathrm{HI})$, the data are Hanning smoothed, resulting in a velocity resolution of $26.4 \mathrm{~km} \mathrm{~s}^{-1}$. Each individual scan was gridded using a combination of aips ++ , aips and miriad. The gridding process, and active scanning method used, introduce some striping into the final images. The gridding also increases the FWHP beamwidth from $14^{\prime} \cdot 4$ to an effective value of $\sim 15^{\prime} \cdot 5$.

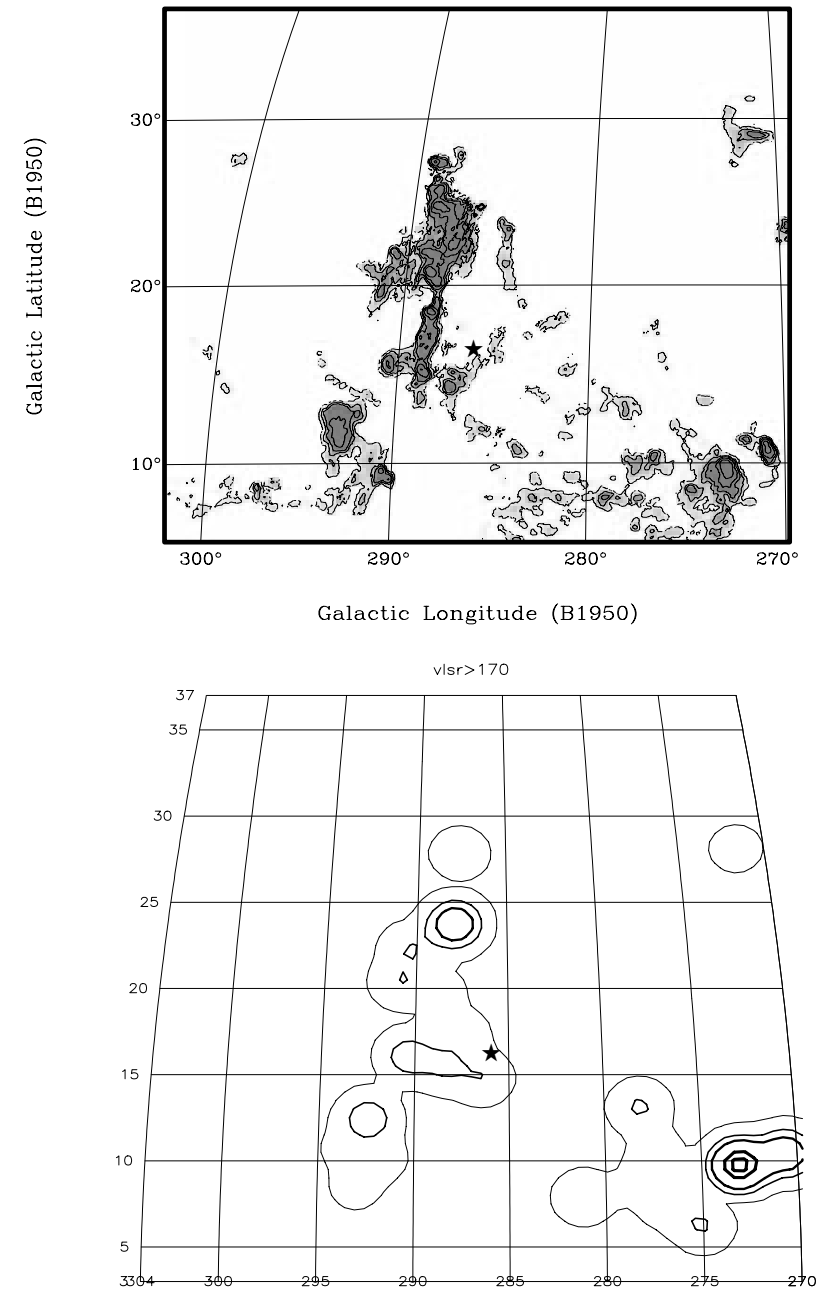

Figure 1-Column density maps of HIPASS data (top) and data from the Bajaja et al. (1985) survey (bottom). The maps cover the same $\sim 1000 \mathrm{deg}^{2}$ region of sky, centred upon the Extreme Positive Population of HVCs (adopting the nomenclature of Wakker \& van Woerden 1991). The velocity range included here is $v_{\mathrm{lsr}}=170-400 \mathrm{~km} \mathrm{~s}^{-1}$. The star symbol represents the position of the Seyfert 1, ESO 265-G23 (see text).

\section{The Multibeam Advantage}

The key parameters of HIPASS are contrasted with the B85 and VESSS southern surveys in Table 1. The primary advantages of HIPASS are its superior spatial resolution and dense spatial sampling. If 


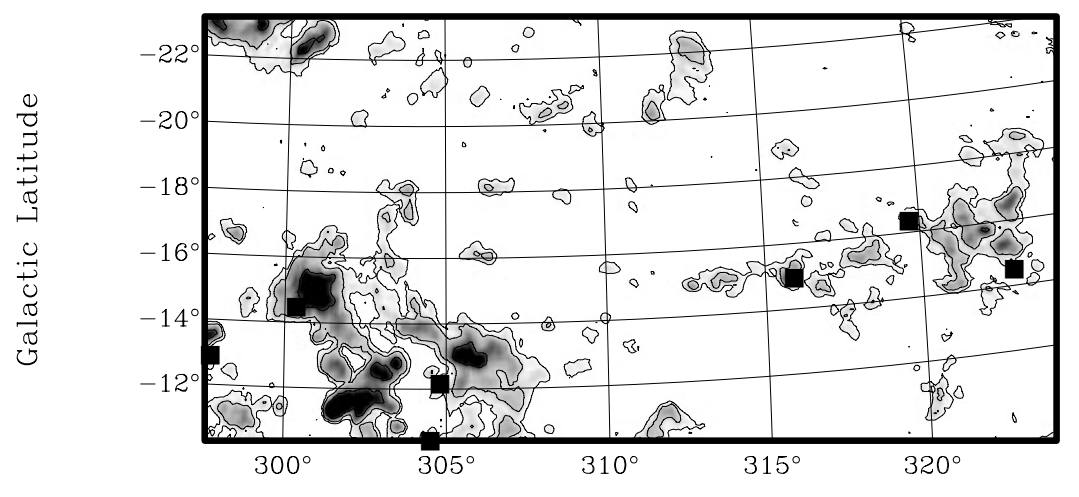

Galactic Longitude

Figure 2-Peak intensity map of positive velocity HVCs $\left(v_{\mathrm{lsr}}=85-400 \mathrm{~km} \mathrm{~s}^{-1}\right)$. The solid boxes represent the positions of all (i.e. 7) clouds in the Wakker \& van Woerden (1991) compilation.

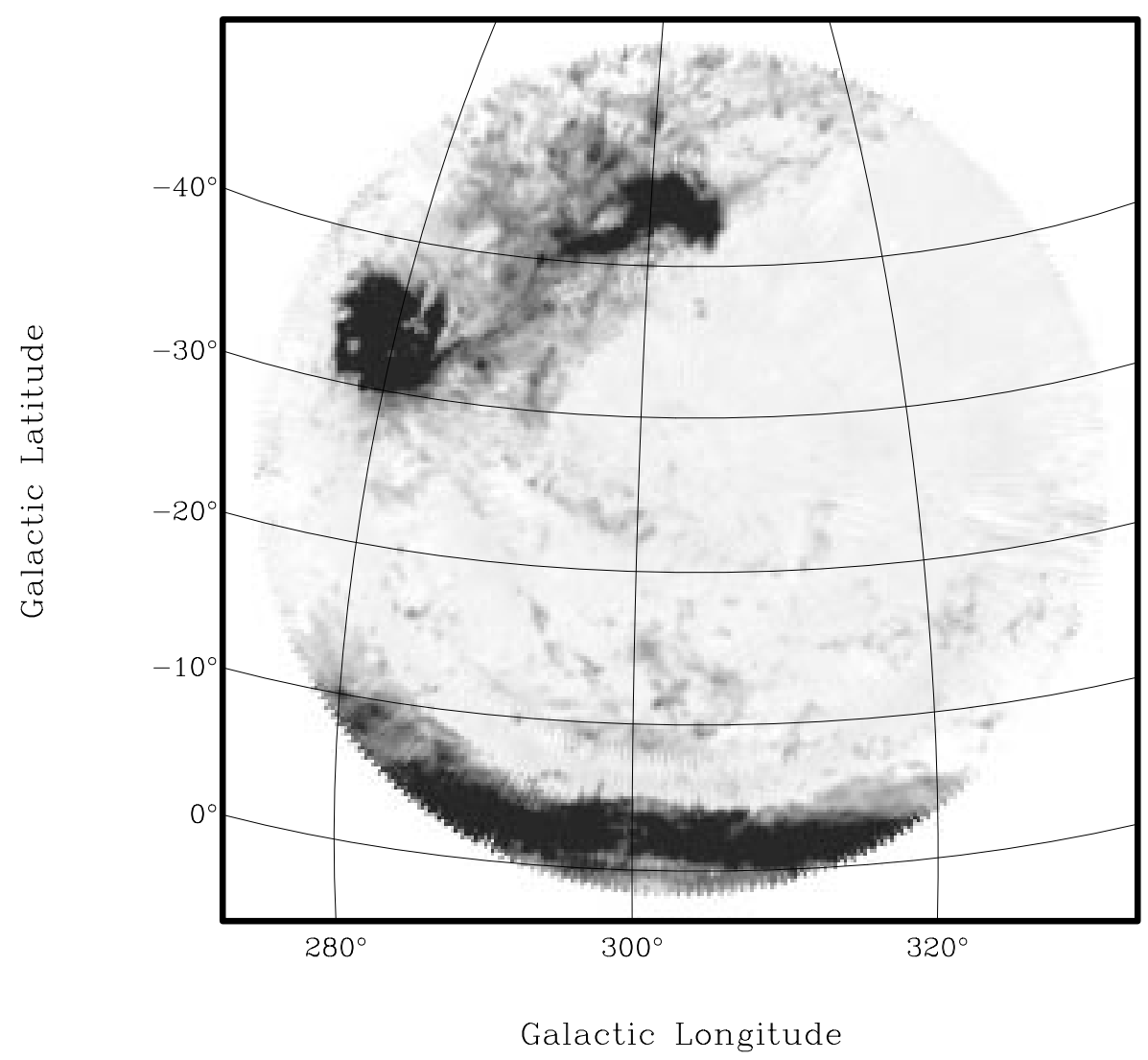

Figure 3-Peak intensity map of a $2400 \mathrm{deg}^{2}$ region centred upon the South Celestial Pole, including the region shown in Figure 2. The velocity range encompassed here is $v_{\mathrm{lsr}}=85-400 \mathrm{~km} \mathrm{~s}^{-1}$. The Large and Small Magellanic Clouds, the Magellanic Bridge and the beginning of the Magellanic Stream are shown at the top of the figure. The Galactic Plane, which extends out to $120 \mathrm{~km} \mathrm{~s}^{-1}$ in the direction $(\ell, b)=\left(300^{\circ}, 0^{\circ}\right)$ (Burton 1988), is seen at the bottom of the figure.

HIPASS's active scanning method was put on a point-and-shoot grid it would correspond to a grid spacing of $\sim 6^{\prime}$ with a $\sim 15^{\prime} .5$ beam. The HIPASS survey zone also covers almost twice as much of the sky as the Argentinian surveys, providing desired consistency checks with the northern hemisphere HVC surveys. Some of the differences alluded to in Table 1 are best expressed graphically. Figure 1 shows the same region of sky as viewed by HIPASS and B85.
Clearly, the gross properties are visible in the B85 data, but just as clear is the startling improvement provided by the HIPASS data. The low resolution and sparse sampling of B85 tends to merge many individual features into a single, amorphous blob; the complex structure of the clouds only becomes apparent when examining the HIPASS data.

We are already in a position to speculate on the expected increase in the number of catalogued, 
isolated, HVCs and CHVCs. Based on a visual inspection of the data in hand, we expect this number to increase by a factor of 5 . This is not to say that the integrated Hi flux or mass will increase by a factor of 5 , but that the number (especially of CHVCs) is expected to increase dramatically. These results will greatly affect $\mathrm{HVC}$ catalog statistics. What previously appeared to be a lack of positive velocity clouds, may simply have been the inability of previous surveys to resolve the individual clumps. Figure 2 offers further evidence to this effect; the boxes therein mark the positions of all previously catalogued clouds in this area (from Wakker \& van Woerden 1991). Again, the large population of individual HVCs missed by B85 (and thus the Wakker \& van Woerden compilation) is clearly evident. We will return to Figure 2 shortly.

Just as important as the discovery of enormous numbers of new clouds is the parallel increase in positional accuracy of existing (and new) HVCs. This has important implications for optical and UV follow-up programs to determine HVC distances and metallicities. Both programs hinge upon knowledge of an HVC's intervening Hi column density along the line of sight to a suitable stellar or extragalactic probe. Basing these programs upon the older, low resolution data is risky, but was in the past a necessity. As alluded to in Figure 1, HIPASS will improve this situation dramatically. Looking at the B85 data, the background Seyfert galaxy ESO 265-G23 was thought to be aligned with the extreme positive HVC shown; the HIPASS data show that it is actually situated in what appears to be an HI-free region. Ultimately, even higher-resolution synthesis observations are needed (a program we are pursuing at the ATCA), but obviously HIPASS offers a superior base to build upon when prioritising candidate background probes. Similar arguments can be made when drawing up target lists for follow-up emission-line studies, a program we are pursuing with J. Bland-Hawthorn at the AAT and WHT (see Bland-Hawthorn et al. 1998).

Another advantageous feature of HIPASS is its unbiased sky coverage. The displayed population of positive HVCs in Figure 2 looks fairly unremarkable when framed within a $\sim 300 \mathrm{deg}^{2}$ region. It is only upon expanding one's view by an order of magnitude that their true nature can be appreciated. The sheer size of the HIPASS dataset makes such 'big picture' views easily attainable. Figure 3 takes the clouds of Figure 2, and places them into the context of a 2400 $\operatorname{deg}^{2}$ area of sky. Only now does the clouds' relationship to the Magellanic System become apparent. Their link to the Magellanic Clouds becomes even more evident in the individual channel maps. Figure 4 shows the channel centred upon $v_{\mathrm{lsr}}=323 \mathrm{~km} \mathrm{~s}^{-1}$ and depicts the feature's spatial continuity for over $25^{\circ}$, starting from a region near the Magellanic Bridge.

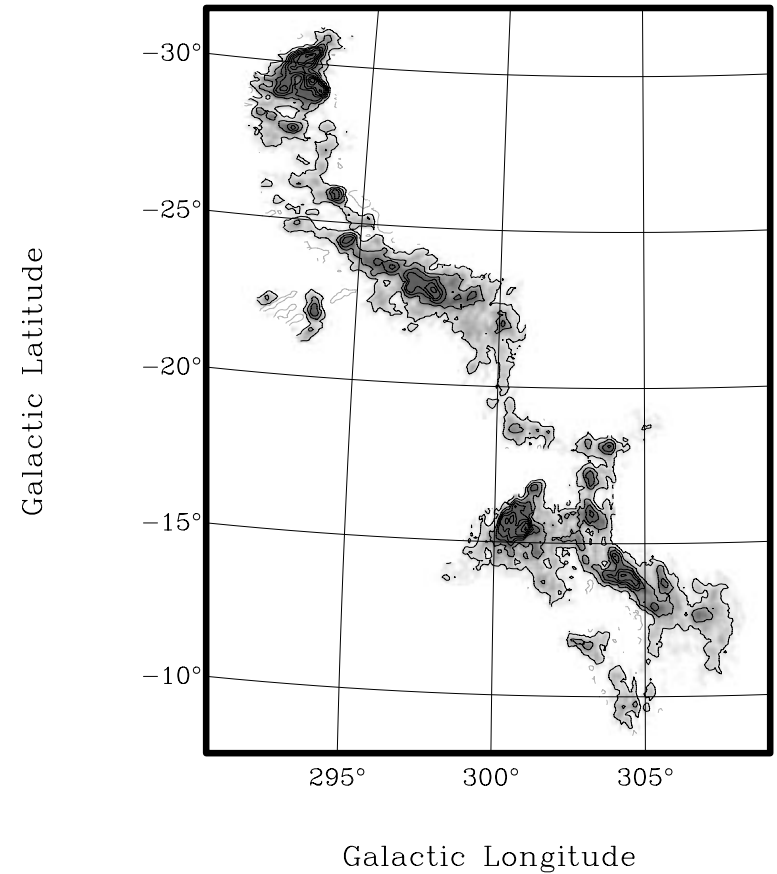

Figure 4-A detailed view of the Leading Arm at the channel centred upon $v_{\mathrm{lsr}}=323 \mathrm{~km} \mathrm{~s}^{-1}$. Contours are from $10-90 \%$ of the brightness temperature maximum $\left(T_{\mathrm{B}}=0 \cdot 88 \mathrm{~K}\right)$. The link between the Magellanic System and the strong emission features at $(\ell, b)=\left(297^{\circ},-24^{\circ}\right)$ and $(\ell, b)=\left(302^{\circ},-16^{\circ}\right)$ is not visible in the earlier data of Mathewson \& Ford (1984) (their Figure 2) or Morras (1982), due to their sparse spatial sampling. The total Hi mass of the Arm is $\sim 1 \times 10^{7} M_{\odot}$. See Putman et al. (1998) for further details.

This continuous feature on the leading side of the Magellanic Clouds, highlighted by Figure 4, is a natural prediction of tidal models which simulate the dynamical interaction of the Clouds with the Milky Way (e.g. Gardiner \& Noguchi 1996). Our discovery of this Leading Arm was reported in Putman et al. (1998); parts of the feature are apparent in the old Mathewson \& Ford (1984) data, but it was only the advent of the HIPASS reductions which allowed us to demonstrate the spatial and kinematic continuity of this feature. The lack of continuity had been used by proponents of ram pressure Magellanic Stream formation models to claim the tidal model was excluded (e.g. Moore \& Davis 1994); on the contrary, our finding of a continuous Leading Arm which emanates from the Magellanic System has allowed us to eliminate virtually all pure ram pressure models.

\section{HVC Structure}

Continuous streamer structure is a common phenomenon which appears in the fully sampled HIPASS data. The Magellanic Stream (which trails for $\sim 100^{\circ}$ behind the Clouds) and Leading Arm, discussed in the previous section, are obvious examples. Another example of a spatially continuous filament was shown in Figure 1; this feature also has a strikingly systematic velocity structure (see Putman \& Gibson 


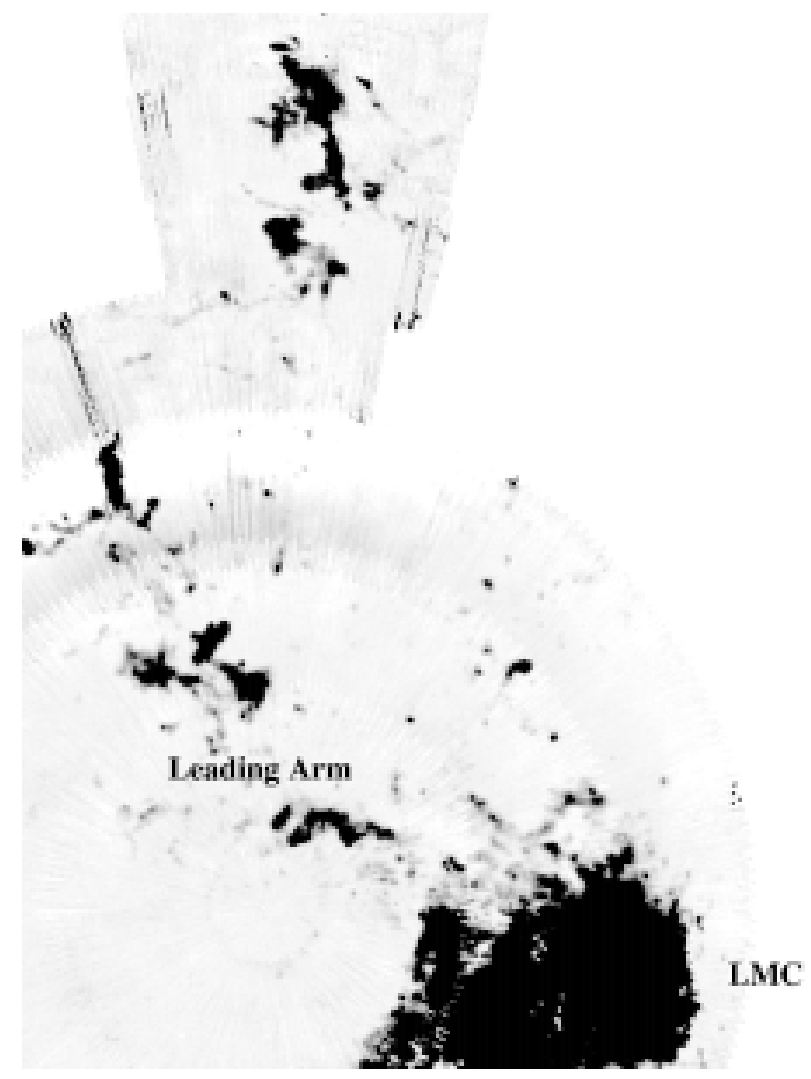

Figure 5-Peak intensity map which includes regions from Figures 1 and 3 , and velocities $v_{\mathrm{lsr}}=165-400 \mathrm{~km} \mathrm{~s}^{-1}$.

1999 or Morras \& Bajaja 1983). Figure 1 (and also Figure 4) can be put into further context by examining Figure 5. Here, the filamentary structure of Figure 1 (shown at the top of Figure 5) appears to be an extension of the Leading Arm shown in Figure 4, the entire structure perhaps representing a pseudo-continuous, $\sim 50^{\circ}$-long, leading tidal feature. Such a scenario is supported by the metallicity determination for the dominant filament in Figure 1 from Lu et al. (1998), which matches that of the Magellanic Clouds. However, the 'kink' in the Leading Arm above the Galactic Plane appears to be difficult to recover under a tidal scenario, as well as in tidal models which incorporate a small degree of drag (Gardiner 1999).

We should stress that while filamentary $\mathrm{HI}$ structure may be common, assigning a tidal origin to all such features would certainly be incorrect. There are many situations where the filamentary HVC actually connects to Galactic emission when it is traced back in position and velocity. This is demonstrated in Figure 6, where the knot of emission in the $v_{\mathrm{lsr}}=86 \mathrm{~km} \mathrm{~s}^{-1}$ channel corresponds to HVC\#350 in the Wakker \& van Woerden (1991) compilation. While technically this is an HVC because of its anomalous velocity, Figure 6 makes it readily apparent that it is not an isolated $\mathrm{HVC}$, but an extension of Galactic emission. This example reiterates an earlier point, that clearly not all HVCs are created by the same mechanism - while HVC\#350 appears to be associated with the Galaxy, those of Figure 5 are almost certainly (predominantly) associated with tidal stripping of material from the Magellanic System. Figure 6 demonstrates that blind adoption of velocity cut-offs in any HVC catalog construction is a dangerous practice which does not necessarily take into account the 'big picture'.

It is tempting to associate stream-like 'HVCs', such as those shown in Figure 6, with the continuation of the worms defined by Koo, Heiles \& Reach (1992), possible supernova break-outs, or the splash-down effects of discrete HVCs. While, as noted already, continuous streams of anomalous $\mathrm{HI}$, such as the Magellanic Stream and its associated Leading Arm, which are clearly separate from Galactic emission, almost certainly reflect the tidal disruption of our neighbours (both present-day and past). Perhaps the overall filamentary structure of the clouds is also related to magnetic fields which extend into the Galactic halo (Kalberla \& Kerp 1998)? Dense clumps of emission, or CHVCs in the nomenclature of Braun \& Burton (1999)*, may represent a distinct category, populating the intergalactic medium of the Local Group, as suggested by Blitz et al. (1999). On the other hand, they often appear to be associated in position and velocity with neighbouring large-

* Braun \& Burton (1999) define a CHVC as an isolated cloud which has an angular size of less than $2^{\circ}$ FWHM. 


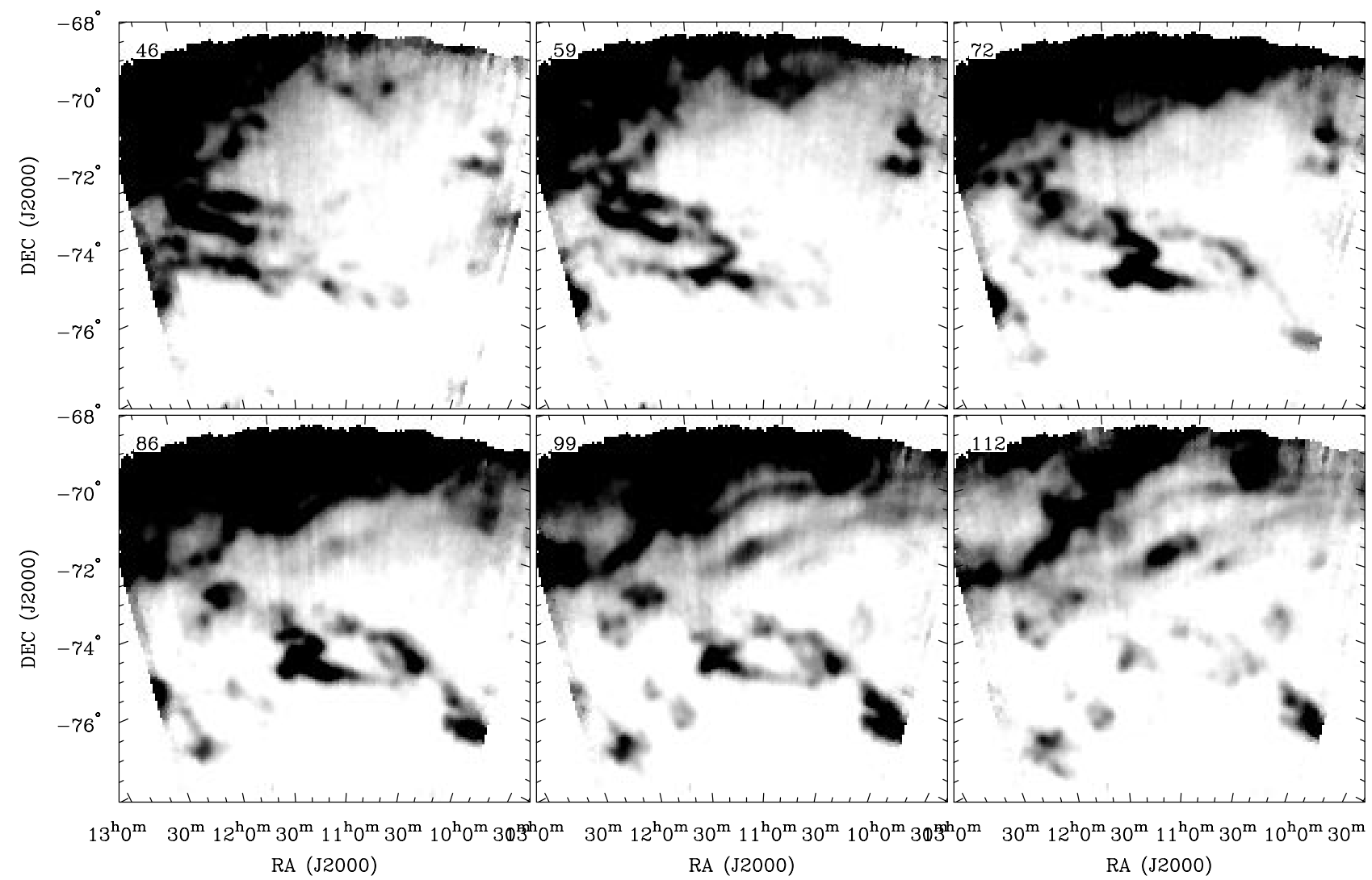

Figure 6-Channel maps showing the HVC designated \#350 in the Wakker \& van Woerden (1991) compilation [particularly evident by the knot of emission in the $v_{\mathrm{lsr}}=86 \mathrm{~km} \mathrm{~s}^{-1}$ channel ( $v_{\mathrm{lsr}}$ is labelled in the upper left of each panel.)]. This is clearly not an isolated HVC, per se, but one whose emission merges with the neighbouring Galactic emission.

scale complexes, and they could simply be small bits of interactive debris, as demonstrated in the hydrodynamical simulations of Li \& Thronson (1998). Mapping the $\mathrm{H} \alpha$ emissivities of these CHVCs, along with their neighbouring complexes, may provide clues as to any differences which might exist in the ionising environments in which they reside.

The complex structure of HVCs defines our need for dense spatial sampling and high resolution observations. As shown here, the Parkes Multibeam HVC Survey has the capability to greatly increase our understanding of HVC distribution and structure, with the Leading Arm feature a perfect example of its success in uncovering new features in the Galactic halo. Categorising clouds according to their spatial and velocity distribution will be a crucial future step for uncovering the origin of various high velocity complexes. We are actively pursuing a program of $\mathrm{H} \alpha$ emission studies, high-resolution $21 \mathrm{~cm}$ synthesis work, and FUSE (Far Ultraviolet Spectroscopic Explorer) and GHRS (Goddard HighResolution Spectrograph) metallicity determinations for a number of HVCs which populate a wide variety of environments. By putting these results into the context of the Parkes Multibeam HVC Survey we should be able to provide key clues to the mysterious origin(s) of HVCs.

\section{Acknowledgments}

We thank the Multibeam Survey Working Group for their help in the HIPASS data collection, and the development of the instrumentation and software. The assistance of Bart Wakker, in preparing part of Figure 1, is likewise acknowledged.

\section{References}

Bajaja, E., et al. 1985, ApJS, 58, 143

Barnes, D. G., Staveley-Smith, L., Ye, T., \& Oosterloo, T. 1998, in Astronomical Data Analysis Software and Systems (ADASS) VII, ed. R. Albrecht et al., ASP Conf. Series, Vol. 145 , p. 89

Bland-Hawthorn, J., Veilleux, S., Cecil, G. N., Putman, M. E., Gibson, B. K., \& Maloney, P. R. 1998, MNRAS, 299, 611

Blitz, L., Spergel, D. N., Teuben, P. J., Hartmann, D., \& Burton, W. B. 1999, ApJ, submitted

Braun, R., \& Burton, W. B. 1999, A\&A, 341, 437

Burton, W. B. 1988, in Galactic and Extragalactic Radio Astronomy, ed. G. L. Verschuur \& K. I. Kellermann (Berlin: Springer), p. 295

Gardiner, L. T. 1999, in Stromlo Workshop on High-Velocity Clouds, ed. B. K. Gibson \& M. E. Putman, ASP Conf. Series, Vol. 166, p. 292

Gardiner, L. T., \& Noguchi, M. 1996, MNRAS, 278, 191

Hartmann, D., \& Burton, W. B. 1997, Atlas of Galactic Neutral Hydrogen, Cambridge Univ. Press

Hulsbosch, A. N. M., \& Wakker, B. P. 1988, A\&AS, 75, 191

Kalberla, P. M. W., \& Kerp, J. 1998, A\&A, 339, 745 
Koo, B., Heiles, C., \& Reach, W. T. 1992, ApJ, 390, 108

Li, P. S., \& Thronson, H. A., 1999, in New Views of the Magellanic Clouds, IAU Symp. 190, ed. Y.-H. Chu et al., ASP Conf. Series, in press

Lu, L., et al 1998, AJ, 115, 162

Mathewson, D. S., \& Ford, V. L. 1984, in Structure and Evolution of the Magellanic Clouds, ed. S. van den Bergh \& K. S. de Boer (Dordrecht: Kluwer), p. 125

Mathewson, D. S., Cleary, M. N., \& Murray, J. D. 1974, ApJ, 190, 291

Moore, B., \& Davis, M. 1994, MNRAS, 271, 209

Morras, R. 1982, A\&A, 115, 249

Morras, R., \& Bajaja, E. 1983, A\&AS, 51131
Morras, R., Bajaja, E., Arnal, E. M., \& Pöppel, W. G. L. 1999, in Stromlo Workshop on High-Velocity Clouds, ed. B. K. Gibson \& M. E. Putman, ASP Conf. Series, Vol. 166, p. 308

Murphy, E. M., Lockman, F. J., \& Savage, B. D. 1995, ApJ, 447,642

Putman, M. E., \& Gibson, B. K. 1999, in Stromlo Workshop on High-Velocity Clouds, ed. B. K. Gibson \& M. E. Putman, ASP Conf. Series, in press

Putman, M. E., Gibson, B. K., Staveley-Smith, L., et al. 1998, Nature, 394, 752

Staveley-Smith, L. 1997, PASA, 14, 111

Wakker, B. P., \& van Woerden, H. 1991, A\&A, 250, 509

Wakker, B. P., \& van Woerden, H. 1997, ARA\&A, 35, 217 